\title{
Deuxième molaire incluse : apports de la reconstruction tridimensionnelle pour le diagnostic et le traitement
}

\section{Second impacted molar: apports of three dimensionnal reconstruction in diagnosis and therapeutic}

\author{
ANIS HASSEN *, FRÉDÉRIC TRUNDE **, RAPHAËL FILIPPI *, ALAIN HUET *, DENIS BOSSARD ***, JEAN-LOUP COUDERT *
}

\section{RÉSUMÉ}

La rétention de la seconde molaire en arrière de la première molaire est une situation rare que l'orthodontiste peut être amené à rencontrer.

Les trois cas présentés dans cette étude ont été examinés et traités à l'aide d'un logiciel de reconstruction tridimensionnelle à partir des coupes numériques obtenues avec scanner à rayons $\mathrm{X}$.

Son utilisation a permis de porter un diagnostic précis et d'établir un plan de traitement détaillé et adapté à chaque cas. (Med Buccale Chir Buccale 2005; 11 : 223-231).

mots clés: deuxième molaire, dent incluse, reconstruction tridimensionnelle, segmentation

\section{SUMMARY}

médecine

buccale

chirurgie

buccale

VOL. $11, \mathrm{~N}^{\circ} 4$ 2005

page 223

The second molar's retention behind first one is an unusual situation; however, the orthodontist has to deal with it when he encounters the problem.

Three cases where seen and treated in this study with the help of a three-dimensional reconstruction software from scanner $X$ 's numerical sections.

Its use has permitted to make a clear diagnosis and to draw up for each case a precise and adapted treatment plan. (Med Buccale Chir Buccale 2005; 11 : 223-231).

key words: second molar, impacted teeth, three dimensional reconstruction, segmentation

\footnotetext{
* Service de Consultations et de Traitements Dentaires - Lyon

${ }^{* \star} \mathrm{CPE}$ - Domaine scientifique la Doua -Lyon

*** Centre de radiologie -Clinique St-Jean - Lyon

Demande de tirés à part: 
médecine

buccale

chirurgie

buccale

VOL. $11, \mathrm{~N}^{\circ} 4$ 2005

page 224
II existe des variations physiologiques pour l'éruption normale de la deuxième molaire:

- Pour Carlos et Gittelsohn [1] l'éruption de la deuxième molaire supérieure survient à 12 ans 2,9 mois pour les garçons et 11 ans 0,7 mois pour les filles. Pour la deuxième molaire inférieure, à 11 ans 11,2 mois pour les garçons et 11 ans 7,4 mois pour les filles.

- Les variations de l'âge d'éruption s'expliqueraient par des différences dans le développement dentaire, mais aussi par la taille de l'espace postérieur disponible [2].

- Pour Villemey et Duclos [3], et Proy et Gautier [4], l'éruption des dents permanentes se produit plus tôt chez les filles sauf pour les premières molaires permanentes.

- Pour la denture permanente, Logan et Kronfeld [in 5] et Thibault-Lambert [in 6] fixent la date d'éruption de la deuxième molaire à 12 ans $+/-6$ mois. Avant de poursuivre, il semble opportun de rappeler le sens des termes inclusion, enclavement et rétention. Selon l'association française de normalisation [7], on doit utiliser le terme inclusion pour « toute dent retenue dans le maxillaire audelà de sa date normale d'éruption et entourée d'un sac péri-coronnaire sans communication avec la cavité buccale ». Dans l'enclavement, le sac péri-coronnaire est ouvert dans la cavité buccale. La rétention correspond à l'état d'une dent qui est empêchée de poursuivre son évolution alors qu'elle dispose encore d'un potentiel évolutif.

Groover et Lorton (1985) [8], à partir des radiographies panoramiques de 5000 recrues, ont étudié la prévalence de la rétention de la deuxième molaire. Le pourcentage de deuxièmes molaires maxillaires retenues par rapport à l'ensemble des dents retenues était de $0,04 \%$ et de $0,003 \%$ pour les deuxièmes molaires mandibulaires.

Dans une publication sur l'inclusion des molaires permanentes, Tricot Blestel (1990) [9] arrive aux mêmes conclusions que Van Trankmann [10] dont l'étude portait sur 14024 patients; il avait trouvé 223 dents incluses dont $0,76 \%$ sont des deuxièmes molaires maxillaires et $1,94 \%$ des deuxièmes molaires mandibulaires.

Valmaseda-Castellon et coll (1990) [11], dans une étude sur les anomalies d'éruption des molaires, rapportent 43 cas de molaires retenues (25 patients) et retrouvent une incidence de $0,08 \%$ pour la deuxième molaire maxillaire et de $0,06 \%$ pour la deuxième molaire mandibulaire. Ces incidences différentes sont probablement dues à des variations inter-ethniques, à la composition des échantillons étudiés ainsi qu'à l'âge des patients.

Pour ces inclusions, il existe plusieurs étiologies: elles sont locales, générales, parfois mixtes et souvent difficiles à discerner. Devant ces difficultés on s'est demandé si la reconstruction tridimensionnelle ne pourrait pas apporter des renseignements complémentaires permettant un diagnostic plus précis pour faciliter la décision thérapeutique.

\section{MATERIEL ET METHODE}

Malgré la relative rareté de cette pathologie, trois cas ont été observés récemment. Les patients étaient de sexe masculin et âgés respectivement de 15, 17 et 24 ans. L'examen initial comportait un examen clinique et un examen radiologique avec une radiographie panoramique numérisée.

\section{Patient 1}

17 et 47 ne sont pas présentes sur l'arcade alors que leurs homologues controlatérales terminent leur éruption. Elles sont en position sousmuqueuse et situées dans la concavité de la face distale des premières molaires homolatérales.18 et 48 sont à l'état de germe. Les racines de 17 et 47 paraissent en fin d'édification mais les apex sont encore ouverts. Le risque d'évolution vers l'ankylose conduit à réaliser un scanner à rayons $\mathrm{X}$, la radiographie panoramique ne donnant aucune information sur la position vestibulo-linguale de ces dents (Fig. 1).

\section{Patient 2}

17,37 et 47 sont en place sur l'arcade tandis que 27 n'a toujours pas fait son éruption. La radiographie panoramique révèle un encombrement important et des dents de sagesse encore à l'état de germes. Elle permet également de noter que 18 est mal orientée et 28 mal positionnée, la superposition de 27 et 28 ; cette surperposition 
ne permet pas d'apprécier l'orientation de la 27, ni sa position vestibulo-linguale, ni son degré d'édification radiculaire. Pour obtenir ces différentes informations il est donc nécessaire des réaliser un scanner à rayons $\mathrm{X}$ (Fig. 2).

\section{Patient 3}

27 est retenue tandis que les autres dents de 12 ans ont éffectué leur éruption.

Elle est en position haute et située entre la racine distale de la 26 et le bombé de la 28 qui parait malpositionnée.

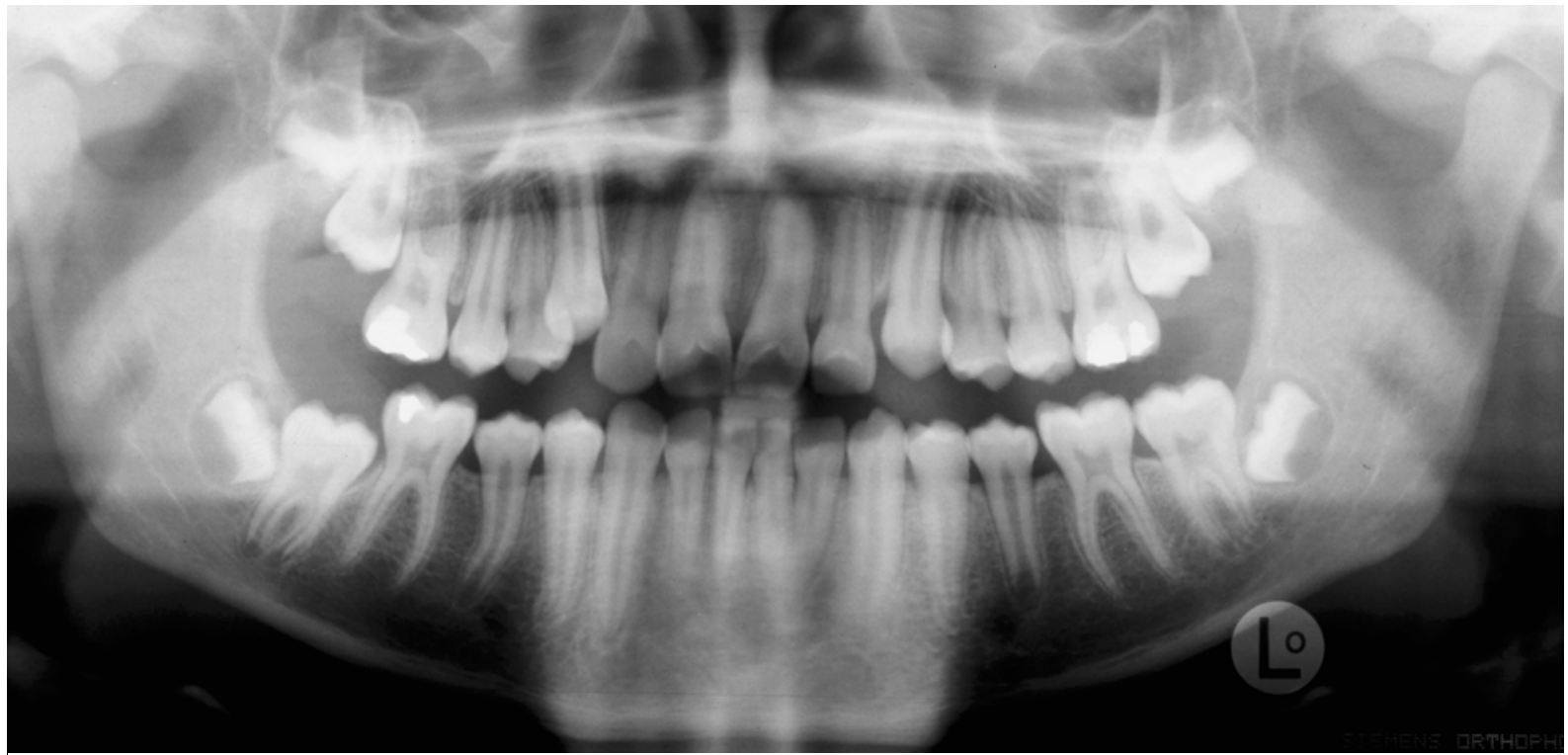

médecine

buccale

chirurgie

buccale

VOL. $11, N^{\circ} 4$ 2005

Figure 1 : Radiographie du patient $\mathrm{n}^{\circ} 1$

Radiography of patient $n^{\circ} 1$

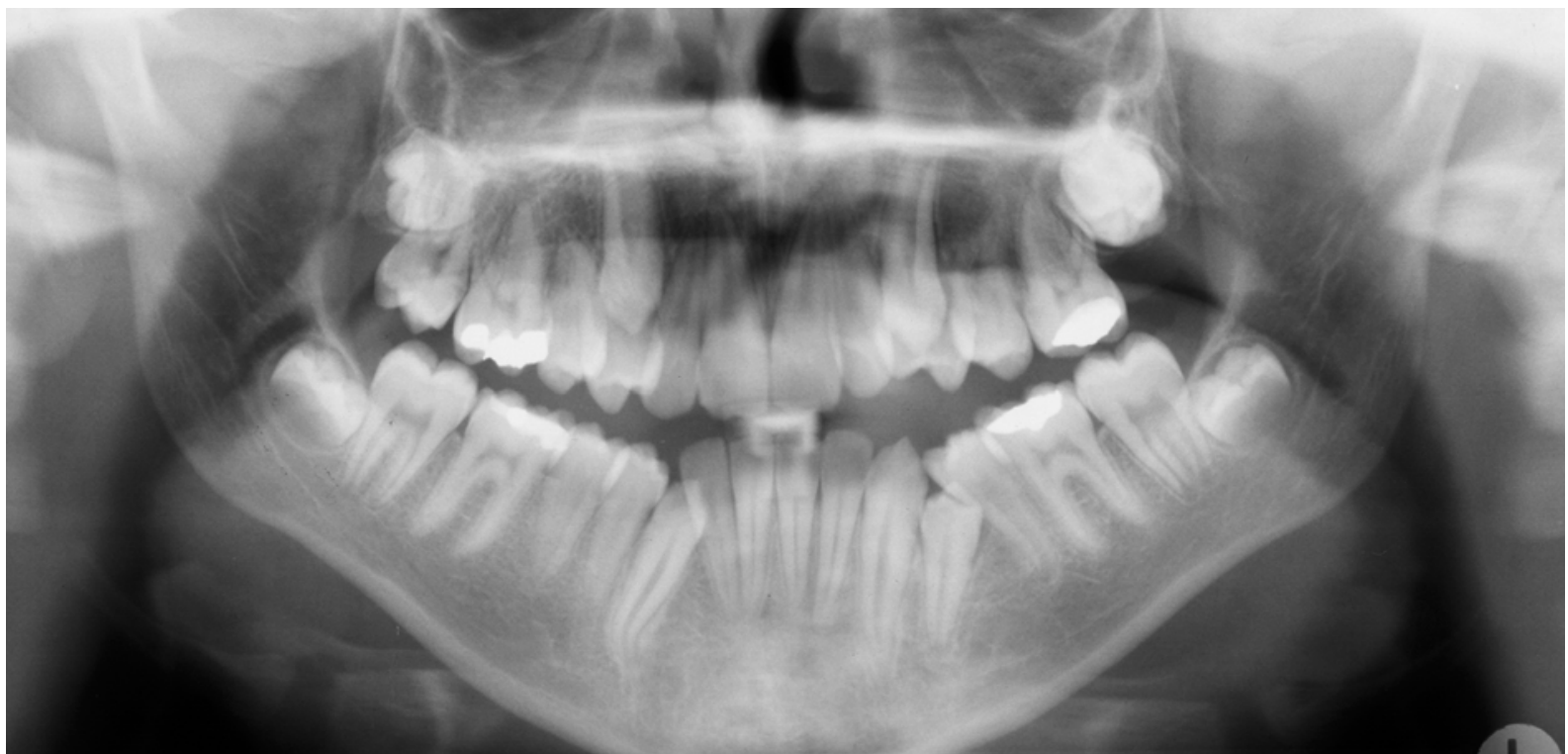

Figure 2 : Radiographie du patient $\mathrm{n}^{\circ} 2$ Radiography of patient $n^{\circ} 2$ 


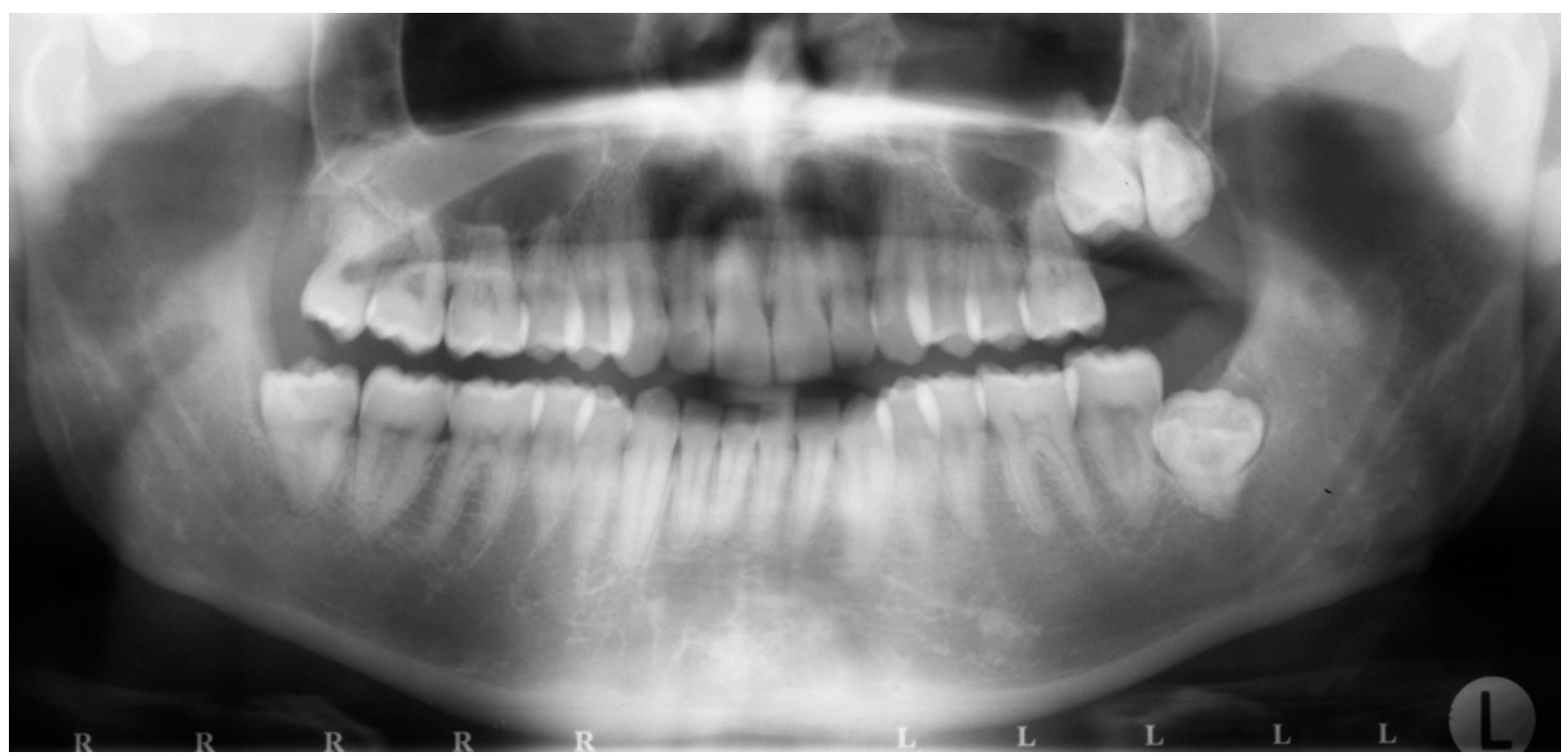

Figure 3 : Radiographie du patient $\mathrm{n}^{\circ} 3$

Radiography of patient $n^{\circ} 3$

médecine

buccale

chirurgie

buccale

VOL. $11, \mathrm{~N}^{\circ} 4$ 2005

page 226
38 est enclavée et son orientation est défavorable.

Là encore un scanner est réalisé afin de déterminer la position exacte de 27 et ses rapports avec les dents qui l'entourent (Fig. 3).

\section{Réalisation des examens scanners à rayons $X$}

Ils sont réalisés avec un appareil de type Somaton + 4 A (Siemens AG, Erlangen, Allemagne). Les paramètres d'acquisition sont de $140 \mathrm{kV}$ et de $129 \mathrm{~mA}$, le filtre de reconstruction fixe à $90 \mathrm{UH}$, le temps de rotation de 1,5 seconde. L'examen est réalisé en acquisition spiralée avec des coupes millimétriques jointives tous les $1 \mathrm{~mm}$.

Lors de l'examen, les maxillaires sont séparés par une compresse sur laquelle mord le patient pour séparer les couronnes anatomiques afin de faciliter la segmentation ultérieure. Le plan de référence est le plan d'occlusion du maxillaire concerné pour limiter les artefacts radiaires dus aux matériaux radio-opaques coronaires.

Le résultat de l'examen scanner à rayons $X$ parvient au praticien sous forme de planches de reprographie argentiques classiques et/ou sous forme d'un CD-ROM.

\section{Création de la scène 3D}

Le logiciel LDS 3D permet une segmentation des différents éléments anatomiques choisis par croissance de région [12]. L'efficacité de cette technique dépend essentiellement du critère de discrimination, de l'appartenance ou non des pixels à la région en construction. Cette tâche est effectuée par une fonction d'évaluation qui détermine le critère d'homogénéité à partir du positionnement initial des germes.

Pour l'étude de 3 cas, la région d'intérêt est limitée aux dents 16,17 et 18 ainsi qu'à l'os de soutien. Chaque élément anatomique est segmenté individuellement, puis ajouté successivement à la scène 3D.

\section{Mesures}

Le logiciel LDS 3D offre la possibilité d'évaluer la distance entre deux points posés sur deux acteurs de la scène 3D. La précision dépend de la valeur du voxel d'acquisition.

\section{Plans}

La création d'un plan est réalisée en définissant 3 points. Pour cette étude, ils sont placés contre la face mésiale de la dent incluse de manière à ce que le plan soit tangent à cette dent. 


\section{RESULTATS}

\section{Patient 1}

Vues de la reconstruction 3D

La couronne anatomique de la 16 est coupée En raison des artefacts radiaires qui rendent la segmentation inopérante (Fig. 4). L'os peut être rendu translucide pour faciliter la lisibilité. (Fig. 5).

\section{Construction du référentiel}

Les 3 points nécessaires à la création du plan de glissement sont placés sur la face mésiale de la dent retenue: les deux premiers sur la ligne de plus grand contour de la couronne et le troisième sur la surface la plus déclive de la racine mésiale (Fig. 6).

Ce plan (plan 1) coupe la couronne de la dent mésiale (16), ce qui montre qu'il existe une gène à l'éruption (Fig. 7). On construit un plan parallèle au précédent (plan 2) passant par un point situé sur la face distale de la 16 et appartenant à la ligne de plus grand contour. La projection du point situé sur la ligne de plus grand contour de la dent 17 en mésial sur le plan 2 permet de mesurer le recul nécessaire pour permettre l'éruption de la dent 17 pour faire son éruption.

Le point choisi sera nommé point mésial, c'est un des points du plan 1, sa projection sur l'autre plan est nommée point projeté (Fig. 7). Sur la vue occlusale, la 17 est bloquée par la 18 en arrière car il existe un contact intime entre ces deux dents; ceci confirme que cette dernière doit bien être extraite (Fig. 8).

La deuxième molaire est placée dans la concavité de la face distale de la 16 et son éruption nécessite un recul de 1,66 $\mathrm{mm}$. La présence de la 18 ne permettant pas un tel recul, elle a été extraite puis un lambeau de repositionnement apical a été réalisé afin de coller un bracket sur la 17. Elle a d'abord été tirée vers l'arrière, puis vers le bas jusqu'à sa mise en occlusion fonctionnelle (Fig. 9).

\section{Patient 2}

Le même référentiel géométrique est mis en place (Fig. 10). La mesure du recul nécessaire pour permettre l'éruption de la 17 est de 5,97 mm. La présence de la dent de sagesse ne permet pas un recul aussi important. De plus la ligne de plus

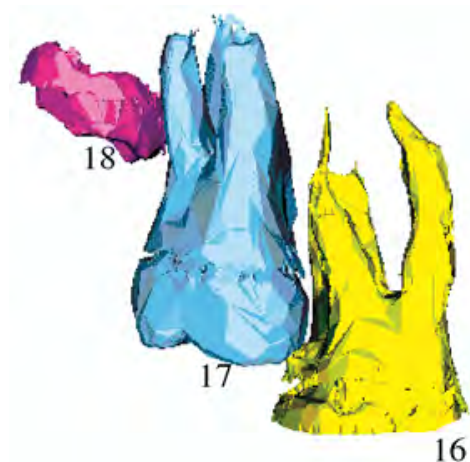

Figure 4 : Dents seules :16,17 et 18 Tooth only: 16,17 and 18.

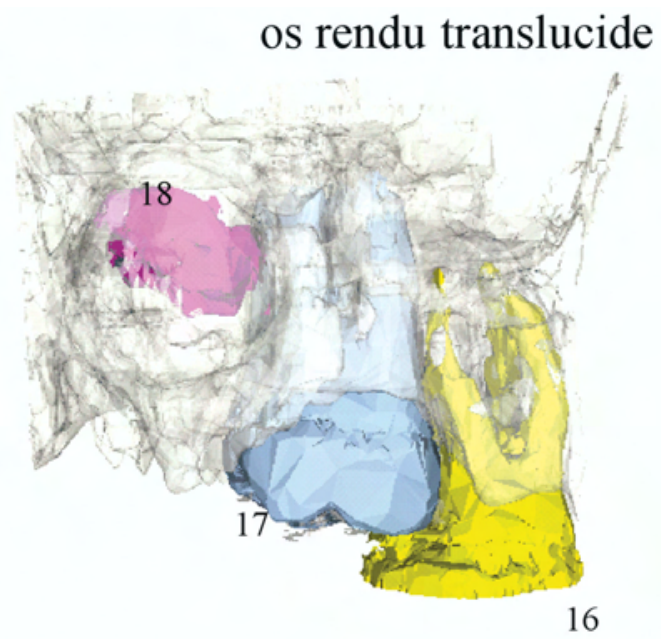

Figure 5 : Scène complète : os + dents Full stage : Bone +Tooth

\section{8}

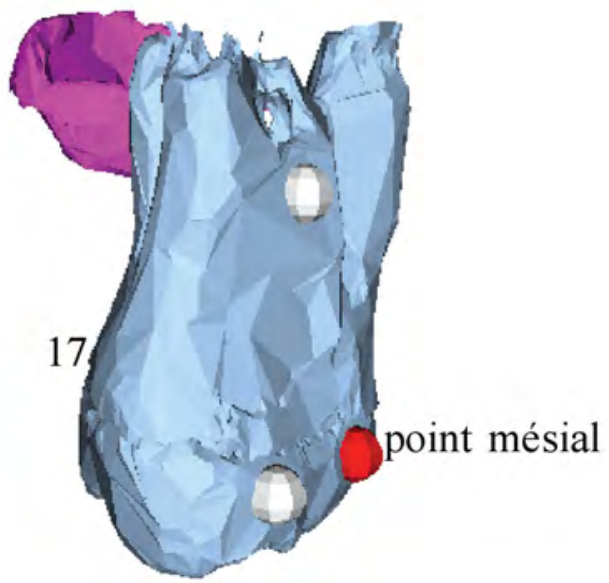

Figure 6 : Construction d'un référentiel - Choix des points Construction of the marks - Point's choice médecine buccale chirurgie buccale

VOL. $11, \mathrm{~N}^{\circ} 4$ 2005

page 227 
médecine

buccale

chirurgie

buccale

VOL. $11, \mathrm{~N}^{\circ} 4$

2005

page 228

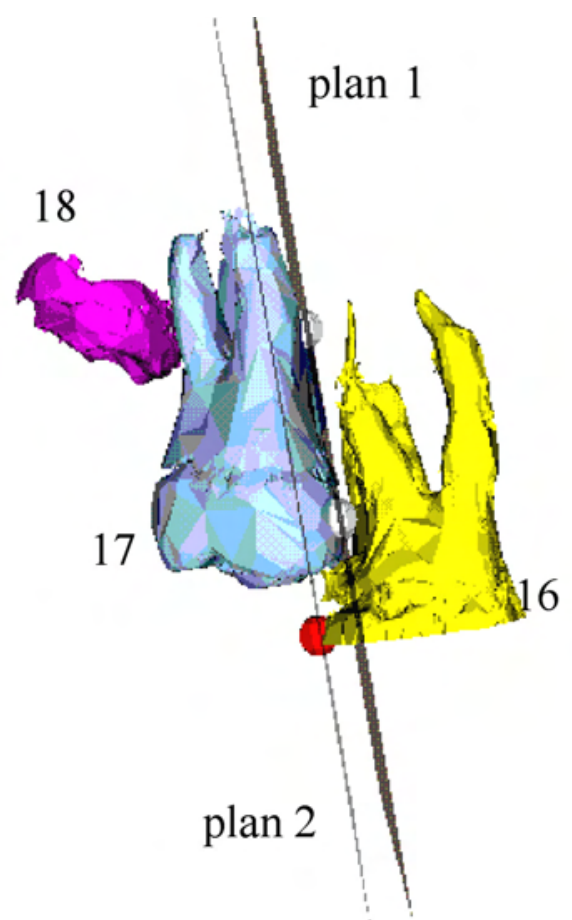

Figure 7 : Construction d'un plan de guidage (Plan 1) et plan parallèle (plan 2)

Construction of a lead plan (Plan 1) and parallel plan (plan 2)

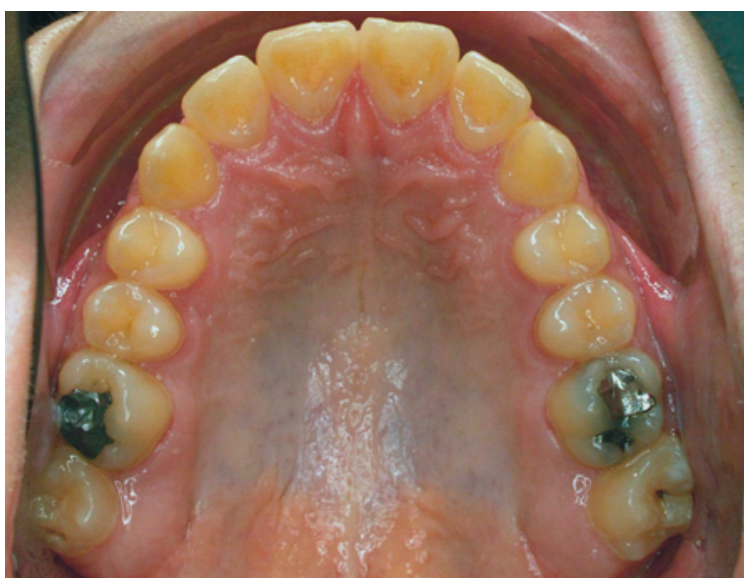

Figure 9 : Patient $\mathrm{n}^{\circ} 1$ : après traitement Photograph $n^{\circ} 1$ : After treatment

grand contour de la 27 est située au-dessus de celle de la 28. Cette dernière doit donc être extraite pour permettre le recul de la deuxième molaire afin d'amorcer sa mise en place sur l'arcade.

\section{Patient 3}

Les mêmes constructions géométriques (Fig. 11) sont à nouveau réalisées pour le calcul des

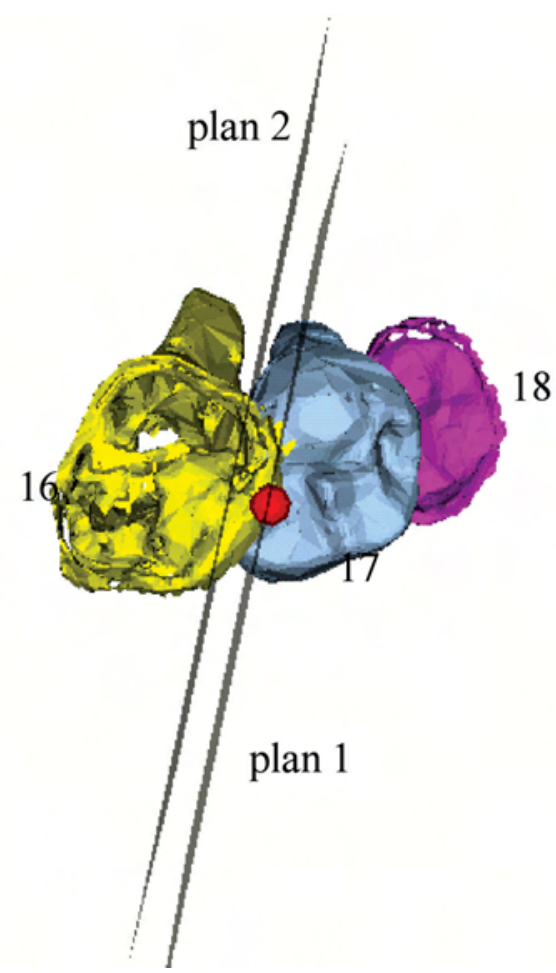

Figure 8 : Vue occlusale Occlusal view

mesures. L'axe de la dent de la deuxième molaire présente une rotation corono-mésiale qui est incompatible avec le plan de glissement de la première molaire.

La première molaire présentait une restauration à l'amalgame donnant d'importants artefacts radiaires gênant la segmentation de la partie coronaire de la dent. Néanmoins, cette vue est beaucoup plus parlante que la radiographie panoramique. Elle permet aussi d'apprécier l'axe de chaque dent.

Dans ces 3 cas, les deuxièmes molaires ont été amenés à l'arcade. Chaque fois l'extraction de la dent de sagesse a été suivie du collage d'un attachement orthodontique sur la face vestibulaire de la deuxième molaire associé à la réalisation d'un lambeau de repositionnement apical. Le traitement orthodontique s'est déroulé en deux temps : la deuxième molaire est d'abord repoussée vers l'arrière puis tractée vers le bas. Les deuxièmes molaires mise en place sont vitales et en occlusion fonctionnelle. 


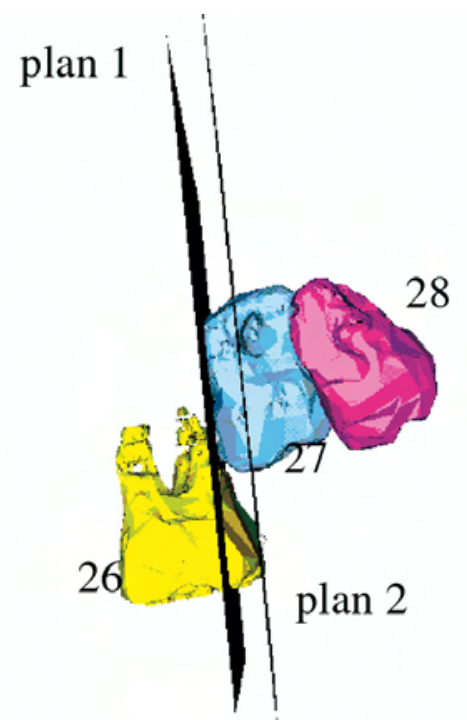

os alvéolaire

Figure 10 : dents + plans et scène complète Tooth + plans and full stage

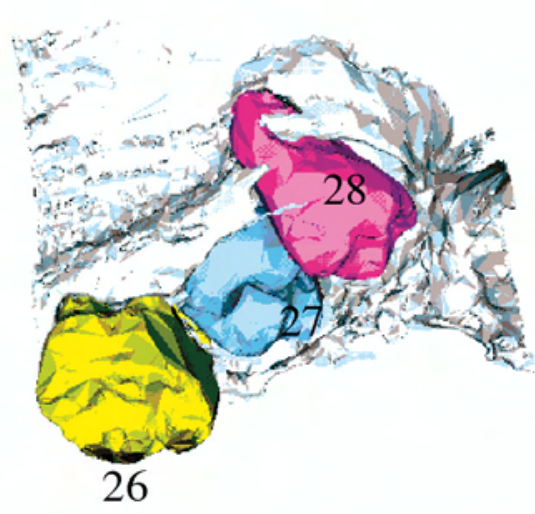

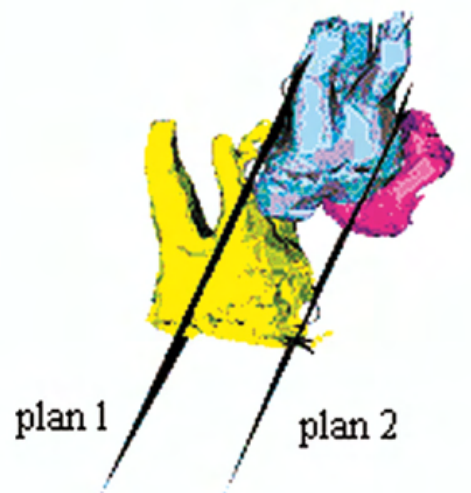

27

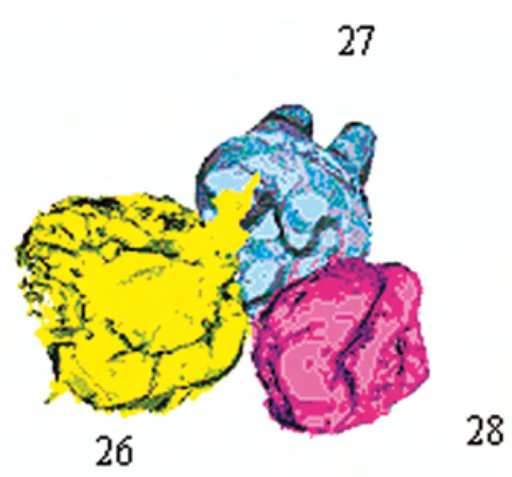

médecine

buccale chirurgie buccale

VOL. $11, \mathrm{~N}^{\circ} 4$ 2005

page 229

Figure 11 : Scène des mesures du patient $n^{\circ} 3$ et vue occlusale Stage of patient 3's measures and occlusal view

\section{DISCUSSION}

\section{Apports pour le diagnostic}

Les informations apportées par cette technique viennent compléter et préciser celles obtenues par l'imagerie classique en 2 dimensions.

Les apports sont qualitatifs:

- La clarté de la scène permet de visualiser la position relative de chaque organe dentaire avec précision et d'observer le rapport des trois molaires entre elles sous l'angle souhaité. Pour certains auteurs, l'inclusion de la deuxième molaire est due à l'évolution de la dent de sagesse. Saive et Mathis [13] suggèrent qu'elle résulte plutôt d'un encombrement. Pour M.C.Tricot Blestel [9], ces facteurs sont associés dans la plupart des cas.

Avec la reconstruction $3 D$, on a pu confirmer que l'inclusion de la deuxième molaire peut être secondaire à un manque de place entre la première molaire et la dent de sagesse.

- La possibilité d'associer tous les acteurs ou au contraire d'en dissocier certains en les rendant 
médecine

buccale

chirurgie

buccale

VOL. $11, \mathrm{~N}^{\circ} 4$ 2005

page 230 translucides constitue un apport majeur. II devient alors possible de déterminer si le chemin d'éruption est bloqué ou non et de définir la nature de l'obstacle. Pour Mugnier et Bordais [14], cet obstacle peut être constitué par un germe surnuméraire, une tumeur, un kyste, ou par la persistance d'une dent temporaire.

- Par rapport à l'imagerie bidimensionnelle, la lecture est aisée, rapide et précise, les couleurs facilitent la dissociation des acteurs et la compréhension de la scène. Ainsi la position et la morphologie de la dent peuvent être déterminés exactement. De même le diagnostic de malposition est facilité.

En effet Thoma cité par Kaban [15] affirme que, lorsqu'un germe est situé à distance de son site normal, la force d'éruption peut s'épuiser prématurément ou être mal dirigée.

O. Saive et Mathis [13] traitent de l'inclinaison du germe comme facteur favorisant la rétention et ajoutent l'excès d'espace entre la 7 et la racine distale de la 6.

Pour le patient 3 l'orientation des plans montre que l'inclinaison des germes est défavorable (Fig. 11).

Les apports sont aussi quantitatifs.

- Cette technique offre la possibilité de mesurer des distances. De ce fait, l'étiologie est définie avec plus de fiabilité et l'indication thérapeutique mieux posée. Dans les trois cas présentés, l'espace n'était jamais suffisant: pour le patient 1 , il fallait distaler la dent retenue de 1,66 mm; pour le patient 2 de $5,97 \mathrm{~mm}$ et pour le patient 3 de $7,68 \mathrm{~mm}$.

\section{Apports pour le traitement}

Il est possible de faire apparaître également certains éléments anatomiques importants du voisinage comme les paquets vasculo-nerveux ou les cavités sinusiennes (Fig 12); ainsi l'existence de communication entre la dent retenue et le sinus s'apprécie facilement. Ces renseignements permettent de guider le geste chirurgical le risque de projeter le germe dans le sinus, de créer une communication, de léser un nerf ou une artère s'en trouve réduit.

Cette imagerie permet de décider de la conservation ou non de la dent de sagesse lorsque celle-

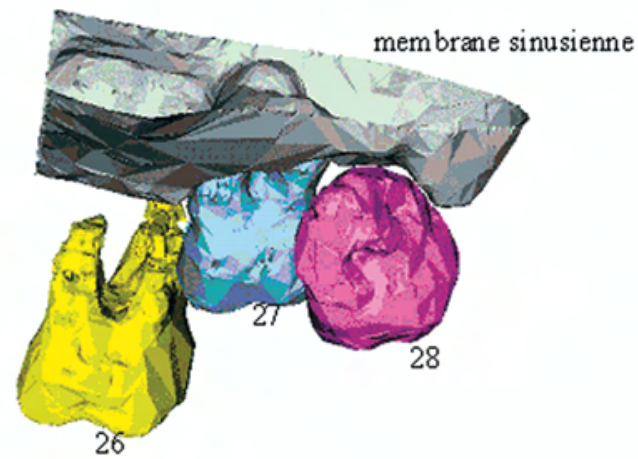

Figure 12 : Rapports sinusiens Connections with the sinus

ci est responsable de la retenue de la deuxième molaire. En mesurant avec précision les distances, on peut confirmer l'importance du manque de place et donc poser d'emblée la bonne indication thérapeutique. On évite ainsi les indications erronées aboutissant à des récidives, de nouvelles perturbations de l'occlusion ou des délabrements osseux importants.

\section{CONCLUSION}

Le scanner est utilisé depuis une dizaine d'années pour l'étude des dents incluses et retenues mais aucun article évoquant de la reconstruction 3D comme outils diagnostique et thérapeutique pour les deuxièmes molaires retenues n'a été trouvé dans la littérature.

L'apport de la reconstruction 3D permet de mieux exploiter les données fournies par le scanner à rayons $X$ en chirurgie buccale et en orthodontie. C'est un outil efficace pour le diagnostic qui offre la possibilité d'affiner le plan de traitement. De plus, il constitue un outil pédagogique dans de nombreuses disciplines.

Toutefois, son utilisation ne peut être systématique. D'abord, cette technique est très chronophage: le temps de segmentation et de préparation de la scène $3 \mathrm{D}$ nécessite 3 heures de travail. D'autre part, le mode d'acquisition impose la prescription d'un scanner à rayons $\mathrm{X}$ qui soulève la question du bénéfice/risque et du coût. Pour le moment, l'indication doit être limitée aux cas complexes. 


\section{RÉFÉRENCES}

1 - Carlos JP, GitTelsohn AM. Eruption patterns of the permanent teeth. J Dent Res 1965; 44 : 509-16

2 - TOMPKINS RL. Human population variability in relative development. Am J Phys Anthropol 1996; 99: 79-102

3 - VILLEMEY A, Duclos P. Age dentaire (6 à 12 ans) - Modes de détermination - Facteurs de variation. Orthod Fr 1971; 42: 1-95

4 - Proy E. Gautier N. L'éruption chez les enfants lyonnais. Rev Orthop Dentofac 1986,a; 20 : 427-41

5 - BÉRARD R. Approche clinique de l'enfant en odontostomatologie, Julien Prélat, Paris 1980.

6 - Bordais P, Gineste P, MARChand J. Accidents de la dentition Encycl Med Chir Paris. Stomatologie, 1977, 22032 A10.

7 - AsSOCIATION FRANÇAISE DE NORMALISATION - Paris, 1968.

8 - GROOVER PS, LORTON L. The incidence of unerupted permanent teeth and related clinical cases Oral Surg Oral Med Oral Pathol 1985; 4 : 420-5

9 - TRICOT BLESTEL MC. L'inclusion des molaires permanentes à l'exception des dents de sagesse. Rev Orthop Dentofac $1990 ; 24$ : 63-80
10 - VAN TRANKMANN J. HaugfigKeit retinierter zähne der zweiten Dentition. Dtsch Zahnärztlz 1973; 28: 415-20.

11 - Valmaseda-Castellon E, De La Rosa-Gay C, Gay-Escoda C. Eruption disturbance of first and second permanent molars: Result of treatment in 43 cases. Am J Orthod Dentofac Orthop 1999; 116 : 651-8

12 - REVol C, JOURLIN M. A new minimum varience region growing algorithm for image segmentation. Pattern Recogn Lett 1997 ; 18 : 249-58

13 - SAIVE O, MATHIS R. Seconde molaire mandibulaire retenue: diagnostic et traitement Actual Odonto, stomatol 2002; 219: 323-32

14 - Coulomb A, Boué D, Vergé F. Dents retenues et anomalies d'éruption. Pedo Fr 1982; 16 : 191-8

15 - Kaban LB, NeEdleman HL, HeRtzBerg J. Idiopathic failure of eruption of permanent molar teeth. Oral Surg Oral Med Oral Pathol 1976; 42: 155-62 \begin{tabular}{l}
$\begin{array}{l}\text { médecine } \\
\text { buccale } \\
\text { chirurgie } \\
\text { buccale }\end{array}$ \\
\hline VOL. $11, N^{\circ} 4$ \\
2005
\end{tabular}

page 231 